\title{
Cerebellar Long Noncoding RNA Expression Profile in a Niemann-Pick C Disease Mouse Model
}

\author{
Shiqian Han ${ }^{1}$ Meng Ren ${ }^{2}$ Tianyin Kuang ${ }^{2} \cdot$ Mao Pang $^{2} \cdot$ Dongwei Guan ${ }^{2} \cdot$ Yesong Liu ${ }^{3} \cdot$ Yong Wang ${ }^{4}$. \\ Wengeng Zhang ${ }^{5}$ Zhijia Ye ${ }^{1,2}$
}

Received: 21 May 2021 / Accepted: 5 August 2021 / Published online: 19 August 2021

(C) The Author(s) 2021

\begin{abstract}
Niemann-Pick type C (NP-C) disease is a neurodegenerative lysosomal storage disorder primarily caused by mutations in $N P C 1$. However, its pathogenesis remains poorly understood. While mounting evidence has demonstrated the involvement of long noncoding RNAs (lncRNAs) in the pathogenesis of neurodegenerative disorders, the lncRNA expression profile in NP-C has not been determined. Here, we used RNA-seq analysis to determine lncRNA and mRNA expression profiles of the cerebella of $\mathrm{NPC}^{-/-}$mice. We found that $272 \mathrm{lncRNAs}$ and $856 \mathrm{mRNAs}$ were significantly dysregulated in NPC1 ${ }^{-/-}$mice relative to controls ( $\geq 2.0$-fold, $p<0.05$ ). Quantitative real-time PCR (qRT-PCR) was utilized to validate the expression of selected lncRNAs and mRNAs. Next, a lncRNA-mRNA coexpression network was employed to examine the potential roles of the differentially expressed (DE) lncRNAs. Functional analysis revealed that mRNAs coexpressed with lncRNAs are mainly linked to immune system-related processes and neuroinflammation. Moreover, knockdown of the lncRNA H19 ameliorated changes in ROS levels and cell viability and suppressed the lipopolysaccharide (LPS)-induced inflammatory response in vitro. Our findings indicate that dysregulated lncRNA expression patterns are associated with NP-C pathogenesis and offer insight into the development of novel therapeutics based on lncRNAs.
\end{abstract}

Keywords Niemann-Pick type C disease · Cerebellum · Long noncoding RNAs · Coexpression network · LncRNA H19

\section{Introduction}

Niemann-Pick type C (NP-C) disease is a rare, autosomal recessive, neurodegenerative lysosomal disorder. Approximately $95 \%$ and $5 \%$ of NP-C cases are caused by mutations in $N P C 1$ and $N P C 2$, respectively [1]. The NPC2 protein

Zhijia Ye

yezj918@cqu.edu.cn

1 Department of Tropical Medicine, College of Preventive Medicine, Army Medical University (Third Military Medical University), Chongqing 400038, China

2 Laboratory Animal Research Center, Chongqing University School of Medicine, Chongqing 400044, China

3 Cornell University, Ithaca, NY 14853, USA

4 Department of Laboratory Animal Science, College of Basic Medical Sciences, Army Medical University (Third Military Medical University), Chongqing 400038, China

5 Precision Medicine Key Laboratory of Sichuan Province and Precision Medicine Center, West China Hospital, Sichuan University, Chengdu 610041, China binds cholesterol released from low-density lipoprotein (LDL) in the lysosome (Lys) lumen and delivers it to the Lys membrane-spanning protein NPC1, which facilitates cholesterol transport to the endoplasmic reticulum and plasma membrane [2-4]. Consequently, NPCl or NPC2 deficiency causes the accumulation of massive amounts of unesterified cholesterol and other lipids (especially glycosphingolipids) in late endosomes (LEs)/Lys [5]. NP-C is characterized by various pathological features in the cerebellum, including the loss of Purkinje cells [6-8]. Its clinical manifestations include progressive neurodegeneration characterized by cerebellar ataxia, dementia, dysphagia, vertical gaze palsy, and gelastic cataplexy [8]. The clinical manifestations and progression of NP-C can be heterogeneous; once neurodegenerative decline has begun, the disease is fatal [1]. To date, how NPC1 protein deficiency impairs brain function, leading to dementia and neurodegeneration, remains unclear. Moreover, clinical treatment agents for NP-C are limited [9]. Thus, a comprehensive understanding of the mechanisms underlying NP-C pathogenesis is urgently required for the development of novel effective therapies. 
Long noncoding RNAs (lncRNAs) are comprised of RNA molecules greater than 200 bases and generally lack protein-coding function [10]. LncRNAs play critical regulatory roles in various biological processes, including genomic imprinting, transcriptional and posttranslational regulation, the maintenance of stem cell pluripotency and the immune response [11]. Numerous studies have implicated dysregulated lncRNAs in neurodegenerative disorders such as Alzheimer's disease [12, 13], autism spectrum disorder [14], Parkinson's disease [15], and Huntington's disease [16], and because of their emerging roles as key modulators of neurodevelopmental pathogenesis, IncRNAs have potential applications as biomarkers or therapeutic targets against neurological disorders $[17,18]$. However, little about lncRNAs and their functional implications in NP-C has been determined.

Here, we used RNA-seq to determine differentially expressed lncRNAs and mRNAs in the cerebella of $\mathrm{NPC} 1^{\text {nih }}$ mice, a well-characterized animal model of NP-C. A lncRNA-mRNA coexpression network was created, and Gene Ontology (GO) and Kyoto Encyclopedia of Genes and Genomes (KEGG) analyses were carried out to predict lncRNA function. Additionally, a NPC1-related coexpression network was constructed to elucidate functional interactions between lncRNAs and NPC1, and the possible mechanisms underlying the development of NP-C were examined.

\section{Materials and Methods}

\section{Animal Models}

$\mathrm{NPC1}^{\text {nih }}\left(\mathrm{NPC1}^{-/-}, \mathrm{KO}\right)$ mice and wild-type (WT) mice were bred from heterozygous pairs of BALB/cNctr-Npc1m1N/J mice (The Jackson Laboratory). The mice were maintained in rooms at a controlled temperature $\left(22-24^{\circ} \mathrm{C}\right)$ and humidity (40-60\%) under a 12-h light/dark cycle. Mouse weight was monitored weekly, and survival time was recorded. Rotarod and coat hanger tests were performed as previously described $[19,20]$, with a minor alteration to the rotarod test; the mice were allowed to remain on the platform for a maximum of $100 \mathrm{~s}$. The cerebella of male $\mathrm{NPC}^{-/-}$mice and WT controls at 7 weeks of age were used for RNA-seq analysis.

\section{Mouse Neurobehavioral Assessment}

Neurobehavioral assessment was performed as described previously [21]. The assessment involved ledge, hind limbclasping, gait, and kyphosis tests. Each measurement was assessed by scoring on a scale of 0-3 (0 indicating no phenotype, 1 indicating a weak phenotype, 2 indicating a strong phenotype, and 3 indicating the most severe phenotype), with a total score ranging from 0 to 12 .

\section{RNA Extraction and RNA-seq Analysis}

Total RNA was isolated from mouse cerebellar tissue using TRIzol reagent (Invitrogen). RNA integrity was determined on an Agilent 2100 Bioanalyzer (Agilent Technologies). RNA purity was determined using a NanoPhotometer spectrophotometer (Implen), and RNA concentration was assessed using a Qubit RNA Assay Kit on a Qubit 2.0 fluorometer (Life Technologies). The RNA samples were then used for RNA-seq and qRT-PCR analyses.

RNA-seq analysis was performed as described previously [22]. Library construction and RNA-seq were performed by Genedenovo Biotechnology Co., Ltd. (Guangzhou, China). The RNA-seq data were normalized based on fragment per kilobase of transcript per million mapped reads (FPKM) by StringTie [23]. DESeq2 program was applied to analyze the differential expression genes between two different groups [24]. Differently expressed (DE) transcripts with a fold change $\geq 2$ and $p<0.05$ in expression were identified by comparison.

\section{qRT-PCR Validation}

qRT-PCR analysis was performed on a CFX96 Real-Time PCR system with the following conditions: $95^{\circ} \mathrm{C}$ for $3 \mathrm{~min}$, followed by 40 cycles of $95{ }^{\circ} \mathrm{C}$ for $5 \mathrm{~s}$ and $60{ }^{\circ} \mathrm{C}$ for $30 \mathrm{~s}$. Relative lncRNA and mRNA expression were determined using the $2^{-\triangle \triangle \mathrm{Ct}}$ approach. GAPDH with no differential expression between two groups in the RNA-Seq profile (Table S1) was used as the reference gene. The primer sequences are provided in Table S2.

\section{GO and KEGG Pathway Analyses}

GO annotation and KEGG pathway analyses were performed to determine the roles of all identified DE mRNAs. GO analysis, which was conducted to annotate the attributes of the genes and gene products, was used to examine enrichment of 3 types of terms in the DE mRNAs: biological process, molecular function, and cellular component terms (http://www.geneontology.org). Pathway analysis was used to determine the biological pathways enriched in the DE genes (http://www.genome.jp/kegg/).

\section{Construction of a Coexpression Network}

To determine the relationships between DE lncRNAs and mRNAs, a coexpression network was built based on the results of correlation assessment of DE lncRNAs and mRNAs [25]. A Pearson's correlation coefficient $\geq 0.980$ and $p<0.05$ were used as cutoffs to determine the DE lncRNAs and mRNAs. To examine interactions between NPC1 and the 
lncRNAs and to predict the functions of related lncRNAs, we identified NPC1-related coexpression networks and used Cytoscape (The Cytoscape Consortium) for visualization.

\section{Small Interfering RNA Transfection}

Skin fibroblasts were isolated from 7-week-old WT and $\mathrm{NPC}^{-1-}$ mice as previously described [26]. Small interfering RNA (siRNA) against mouse H19 (5'-GCAGAATGG CACATAGAAA-3') and control siRNA were synthesized by RiboBio (Guangzhou, China). The skin fibroblasts were transfected with $50 \mathrm{nM}$ si-H19 or control siRNA by electroporation using a NEPA21 electroporator (Nepa Gene) according to the manufacturer's recommendations. Fortyeight hours after electroporation, the cells were harvested and used in the following assays.

\section{Cell Viability Assay}

Cell viability was assessed using the Cell Counting Kit-8 (CCK-8, Biyuntian Biotechnology, Jiangsu, China) assay. Mouse skin fibroblasts were seeded into 96-well microplates at a density of $5 \times 10^{3}$ cells/well after electroporation. Cell viability was assessed by incubating each well with $100 \mu \mathrm{L}$ of CCK- 8 solution for $4 \mathrm{~h}$ after $48 \mathrm{~h}$ of culture under the designated conditions $\left(37^{\circ} \mathrm{C}\right.$ and $5 \% \mathrm{CO}_{2}$ ), and the absorbance at $450 \mathrm{~nm}$ was measured.

\section{Measurement of Intracellular ROS}

Intracellular ROS were detected using the cell-permeant dye CM- $\mathrm{H}_{2}$ DCFDA (Invitrogen). At $48 \mathrm{~h}$ after electroporation, fibroblasts were trypsinized, incubated with $5 \mu \mathrm{mol} / \mathrm{L}$ $\mathrm{CM}-\mathrm{H}_{2}$ DCFDA for $30 \mathrm{~min}$ at $37^{\circ} \mathrm{C}$, and washed twice with PBS. Subsequently, the intracellular formation of ROS was determined by measuring fluorescence with 488 -nm excitation and 538-nm emission wavelengths using a BD Accuri C6 flow cytometer. Relative ROS levels are expressed as the mean fluorescence intensity.

\section{Lipopolysaccharide-Induced Inflammation Assay}

Mouse skin fibroblasts after electroporation were seeded at $5 \times 10^{4}$ cells/well in 12-well plates to incubate for $48 \mathrm{~h}$, and then the cells were stimulated with $100 \mathrm{ng} / \mathrm{mL}$ lipopolysaccharide (LPS) for $24 \mathrm{~h}$ to trigger an inflammatory reaction and collected for RT-PCR.

\section{Statistical Analyses}

All data are indicated as the mean \pm standard error of the mean (SEM). Student's $t$-test was employed to compare the control and experimental groups. One-way analysis of variance (ANOVA) was used to analyze three or more groups. Statistical analyses were done using SPSS 20.0. $p<0.05$ indicated statistical significance.

\section{Results}

\section{$\mathrm{NPC1}^{-/-}$Mice Showed Cerebellar Phenotypes}

Relative to WT littermates, $\mathrm{NPC}^{-1-}$ mice were smaller at weaning and exhibited an initial weight loss at 7 weeks of age (Fig. 1A), decreased rotarod time (Fig. 1B), impaired coat hanger performance (Fig. 1C), and a shortened life span of $65.3 \pm 3.6$ days (Fig. 1D). These pathological phenotypes are consistent with previous reports [27]. To evaluate the relationship between neurological phenotype and onset time in $\mathrm{NPCl}^{-/-}$mice, we used a composite phenotype scoring system based on sensitive and rapid quantification of disease severity [21]. The $\mathrm{NPC1}^{-/-}$mice exhibited progressive cerebellar ataxia starting at 5 weeks of age (Figs. 1E, S1).

\section{Overview of the IncRNA-seq and mRNA-seq Data}

RNA-seq analysis of lncRNA and mRNA expression levels in the cerebella of $\mathrm{NPC}^{-/-}$and WT mice generated $431,617,074$ raw reads. Of these, $218,165,940$ raw reads were from the WT mice and 213,451,134 were from the $\mathrm{NPC}^{-/-}$mice. Upon discarding low-quality sequences, adapter sequences, or sequences for which poly-N $>10 \%$, $430,631,162$ clean reads remained. Of these, 217,672,324 were from the WT mice and 212,958,838 were from the $\mathrm{NPC}^{-1-}$ mice. Next, we conducted a comparative analysis based on paired-end clean reads in the reference genome using HISAT2 [28]. Coding-Non-Coding-Index (CNCI) (v2) [29] and Coding Potential Calculator (CPC) [30] were used to exclude (filter out) transcripts with predicted coding potential. A total of 30,779 lncRNAs $(30,196$ known lncRNAs and 583 novel lncRNAs) and 22,287 proteincoding transcripts (mRNAs) were then used for subsequent analyses. The identified lncRNAs included bidirectional, intergenic, intronic, antisense-overlapping, and sense-overlapping lncRNAs (Figure S2).

\section{DE IncRNAs and mRNAs in NPC1 ${ }^{-1-}$ Mice Compared to WT Mice}

Overall, $272 \operatorname{lncRNAs}$ and 856 mRNAs were found to be remarkably dysregulated in the $\mathrm{NPC}^{-/-}$mice (fold 
A

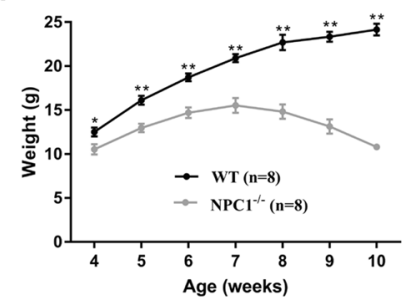

B

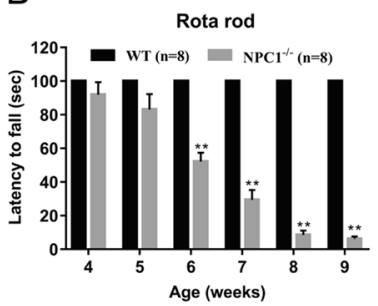

C

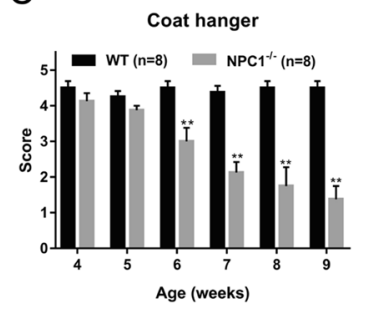

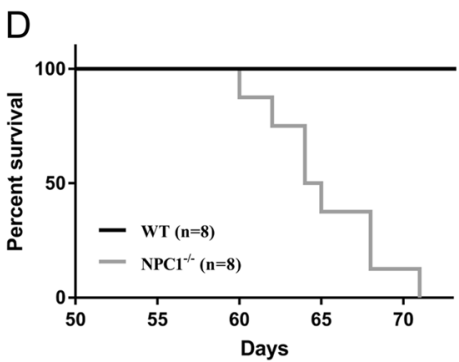

Fig. 1 Cerebellar phenotypes following NPC1 knockout. A Comparison of the body weights of WT and $\mathrm{NPC1}^{-/-}$mice. B Rotarod performance of WT and $\mathrm{NPC}^{-l-}$ mice. C Coat hanger assessment of WT and $\mathrm{NPC}^{-/-}$mice. D Survival analysis of $\mathrm{WT}$ and $\mathrm{NPC}^{-/-}$mice. E Composite phenotype assessment of WT and $\mathrm{NPC1}^{-1-}$ mice. The $\mathrm{NPC}^{-I-}$ mice exhibited a progressive phenotype that was markedly

change $\geq 2.0, p<0.05$ ). Of these, 160 lncRNAs and 682 mRNAs were significantly upregulated, while 112 lncRNAs and 174 mRNAs were significantly downregulated in the $\mathrm{NPC}^{-1-}$ mice versus the WT mice. Thirty lncRNAs and 4 mRNAs were exclusively expressed in the WT mice, while 55 lncRNAs and 10 mRNAs were exclusively expressed in the $\mathrm{NPC}^{-/-}$mice. When the $\mathrm{NPC}^{-/-}$mice were compared with the WT mice, the most significantly upregulated lncRNAs and mRNAs were Trem2 (14-fold change) and Clec7a (36-fold change), while the most significantly downregulated lncRNAs and mRNAs were Eps812 (8-fold change) and Svil (9-fold change). The top 10 most significantly DE lncRNAs and mRNAs are shown in Tables 1 and 2, respectively. Volcano plots and cluster analyses indicated that the lncRNA and mRNA expression patterns were variable and distinguishable between the groups of mice (Fig. 2A-D).

\section{Validation of Gene Expression Profiles by qRT-PCR}

To verify the reliability and accuracy of the differential expression profiles identified by RNA-seq, 5 lncRNAs (Trem2, D430036J16Rik, Rian, Prdx6, and Eps812) and 5 mRNAs (cd68, pckcg, rab32, calb1, and apoe) were randomly selected for qRT-PCR validation. All selected lncRNA and mRNA transcripts were detected with significantly different expressed by qRT-PCR, consistent with the RNA-seq data (Fig. 3A-B).

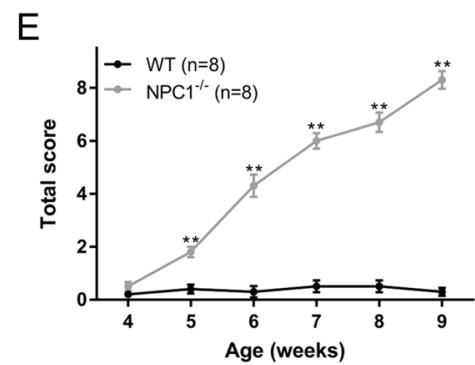

different from the corresponding phenotype in WT mice beginning at 5 weeks. Mice were subjected to ledge, clasping, gait, and kyphosis tests, and performance in each test was scored on a scale of $0-3$. The average composite scores for each genotype at different ages were calculated. Bars represent SEMs. ${ }^{*} p<0.05$ and ${ }^{*} p<0.01$, compared with the WT group

\section{IncRNA-mRNA Network Analysis in NP-C}

To elucidate the potential roles of key lncRNAs and interactions between DE IncRNAs and mRNAs, we built a IncRNA-mRNA coexpression network based on a previously described analytical strategy [31]. The coexpression network consisted of 970 network nodes and 3318 correlations between 738 DE mRNAs and 232 DE lncRNAs. The network contained 725 negative and 2594 positive interactions, suggesting that a single lncRNA can interact with multiple mRNAs and vice versa (Table S3). The DE lncRNAs were mainly distributed on chromosomes 2,7 , and 4 (Fig. 4A), while the DE mRNAs were mainly distributed on chromosomes 11, 6, and 1 (Fig. 4A). The internal connections on the Circos diagram indicate the top 100 strongest correlations between lncRNAs and mRNAs.

Mounting evidence indicates that impaired lysosomal function and calcium distribution in the cellular reticular network mediate NP-C pathogenesis [32, 33]. Thus, Lys- and calciumrelated genes were incorporated into the coexpression network (Fig. 4B-C). Potential interactions between lncRNAs and mRNAs appear to mediate the development of NP-C.

\section{GO and KEGG Pathway Analyses}

Next, we carried out GO and KEGG pathway analyses of the $m R N A s$ in the coexpression network. The terms enriched in a given IncRNA indicated its predicted biological functions. GO analysis 
Table 1 Top 10 upregulated and 10 downregulated lncRNAs

\begin{tabular}{|c|c|c|c|c|c|c|c|c|c|c|}
\hline Ensembl gene ID & Symbol & WT-1 & WT-2 & WT-3 & KO-1 & KO-2 & $\mathrm{KO}-3$ & Fold change & $p$ value & $\overline{\text { Regulation }}$ \\
\hline ENSMUST00000148545 & Trem2 & 3.7004 & 1.0000 & 1.0000 & 6.7142 & 4.8580 & 6.5392 & 14.3121 & 0.0000029 & Up \\
\hline ENSMUST00000142962 & Gm15631 & 2.8074 & 3.1699 & 1.0000 & 5.2095 & 4.9069 & 6.4594 & 10.0690 & 0.0000889 & Up \\
\hline ENSMUST00000031975 & Clec5a & 1.0000 & 2.5850 & 1.0000 & 5.3219 & 4.0000 & 5.1699 & 9.9600 & 0.0001768 & Up \\
\hline ENSMUST00000195685 & A330015K06Rik & 3.5850 & 3.3219 & 3.4594 & 6.6724 & 4.7549 & 6.1898 & 6.2609 & 0.0000273 & Up \\
\hline ENSMUST00000136359 & H19 & 4.3219 & 4.8580 & 5.9069 & 8.3264 & 7.3219 & 6.5236 & 5.4498 & 0.0000664 & Up \\
\hline ENSMUST00000166109 & Eci2 & 2.3219 & 2.5850 & 2.0000 & 4.9542 & 4.1699 & 4.7549 & 5.4028 & 0.0015623 & Up \\
\hline ENSMUST00000131025 & Uap111 & 3.8074 & 2.0000 & 2.5850 & 5.7004 & 4.5850 & 5.4594 & 5.3537 & 0.0009304 & Up \\
\hline ENSMUST00000128338 & Btbd11 & 3.8074 & 1.0000 & 3.5850 & 5.2854 & 5.2095 & 5.1699 & 4.5405 & 0.023154 & Up \\
\hline ENSMUST00000235045 & Gm2629 & 3.5850 & 4.0875 & 3.8074 & 5.9307 & 5.0444 & 6.3750 & 4.1694 & 0.0001185 & Up \\
\hline ENSMUST00000135230 & Copg2 & 6.8455 & 5.2479 & 4.7549 & 7.1898 & 7.4998 & 7.7748 & 3.3397 & 0.0011418 & Up \\
\hline ENSMUST00000155729 & Eps812 & 6.0000 & 6.3219 & 5.9542 & 3.5850 & 2.8074 & 2.3219 & 7.9545 & 5.34E-08 & Down \\
\hline MSTRG.20510.2 & - & 7.1898 & 7.9715 & 6.1293 & 2.0000 & 4.5236 & 4.8580 & 7.7788 & $1.06 \mathrm{E}-06$ & Down \\
\hline ENSMUST00000126572 & Gm13944 & 4.3219 & 5.8580 & 4.3219 & 1.0000 & 2.8074 & 2.3219 & 6.7802 & 0.0011752 & Down \\
\hline ENSMUST00000138576 & Fgf7 & 5.9773 & 6.6147 & 5.8580 & 4.3219 & 3.5850 & 2.0000 & 5.5377 & 9.37E-05 & Down \\
\hline ENSMUST00000238391 & Rian & 5.3219 & 7.4594 & 5.3576 & 4.6439 & 1.0000 & 4.4594 & 5.5229 & 0.0090995 & Down \\
\hline ENSMUST00000152283 & Gipc2 & 5.1293 & 4.8580 & 5.5236 & 2.8074 & 2.3219 & 3.0000 & 5.3684 & 0.0001263 & Down \\
\hline ENSMUST00000139218 & Gm16201 & 6.2288 & 6.1699 & 6.2479 & 3.7004 & 3.7004 & 3.8074 & 5.2619 & 4.16E-08 & Down \\
\hline ENSMUST00000150330 & Necap2 & 5.2095 & 4.9542 & 5.1699 & 1.5850 & 2.8074 & 3.3219 & 5.2566 & 0.0003048 & Down \\
\hline ENSMUST00000181447 & D430036J16Rik & 5.2479 & 5.6439 & 5.3219 & 2.0000 & 3.1699 & 3.4594 & 4.9706 & $6.52 \mathrm{E}-05$ & Down \\
\hline MSTRG.13285.1 & - & 9.2715 & 9.8611 & 9.3729 & 6.0000 & 7.6582 & 7.1599 & 4.9444 & $2.11 \mathrm{E}-09$ & Down \\
\hline
\end{tabular}

Table 2 Top 10 upregulated and 10 downregulated mRNAs

\begin{tabular}{|c|c|c|c|c|c|c|c|c|c|c|}
\hline Ensembl gene ID & Gene symbol & WT-1 & WT-2 & WT-3 & KO-1 & $\mathrm{KO}-2$ & $\mathrm{KO}-3$ & Fold change & $p$ value & Regulation \\
\hline ENSMUSG00000079293 & Clec7a & 3.0000 & 4.9069 & 3.4594 & 9.3859 & 7.9189 & 9.2621 & 35.5133 & $3.08 \mathrm{E}-26$ & Up \\
\hline ENSMUSG00000024672 & Ms4a7 & 1.5850 & 2.0000 & 2.0000 & 7.2854 & 6.0444 & 6.9307 & 29.8605 & $1.05 \mathrm{E}-17$ & Up \\
\hline ENSMUSG00000018774 & Cd68 & 4.3219 & 3.7004 & 3.8074 & 9.0848 & 7.7814 & 9.1472 & 27.0776 & $1.52 \mathrm{E}-39$ & Up \\
\hline ENSMUSG00000038147 & $\mathrm{Cd} 84$ & 5.3576 & 5.6724 & 5.5850 & 9.9410 & 8.7879 & 9.9773 & 22.1628 & $8.68 \mathrm{E}-46$ & Up \\
\hline ENSMUSG00000069516 & Lyz2 & 8.5584 & 8.8106 & 8.4757 & 13.3692 & 11.8234 & 13.1991 & 20.4264 & 4.62E-51 & Up \\
\hline ENSMUSG00000035273 & Hpse & 2.5850 & 5.0000 & 4.0875 & 8.8826 & 7.3309 & 8.7347 & 19.5488 & $3.84 \mathrm{E}-16$ & Up \\
\hline ENSMUSG00000071713 & Csf2rb & 4.1699 & 3.4594 & 2.8074 & 6.6582 & 6.0224 & 6.8580 & 19.3250 & $1.58 \mathrm{E}-13$ & Up \\
\hline ENSMUSG00000050335 & Lgals3 & 4.5236 & 5.2479 & 4.8580 & 9.6073 & 8.1699 & 8.9773 & 17.4630 & $1.63 \mathrm{E}-28$ & Up \\
\hline ENSMUSG00000004552 & Ctse & 3.9069 & 4.1699 & 2.5850 & 8.0279 & 7.0661 & 7.5157 & 15.9878 & $9.87 \mathrm{E}-22$ & Up \\
\hline ENSMUSG00000040552 & C3ar1 & 5.4263 & 5.8074 & 5.6439 & 9.7715 & 8.5584 & 9.5058 & 13.6313 & $1.45 \mathrm{E}-35$ & Up \\
\hline ENSMUSG00000024236 & Svil & 6.1699 & 7.3663 & 8.1997 & 2.3219 & 4.8074 & 3.9069 & 9.0652 & 4.60E-07 & Down \\
\hline ENSMUSG00000041261 & Car8 & 13.7355 & 14.6258 & 14.0700 & 9.9054 & 11.6041 & 10.6671 & 8.8879 & 3.06E-06 & Down \\
\hline ENSMUSG00000041476 & Smpx & 5.8074 & 6.3923 & 6.0224 & 2.0000 & 3.5850 & 2.3219 & 7.6154 & $2.30 \mathrm{E}-08$ & Down \\
\hline ENSMUSG00000027208 & Fgf7 & 7.4838 & 8.9218 & 8.4471 & 5.0000 & 5.6147 & 4.4594 & 7.5887 & $2.28 \mathrm{E}-11$ & Down \\
\hline ENSMUSG00000002930 & Ppp1r17 & 11.0573 & 11.5920 & 11.0471 & 7.4676 & 8.9687 & 8.2240 & 6.7945 & $4.90 \mathrm{E}-07$ & Down \\
\hline ENSMUSG00000027296 & Itpka & 9.2574 & 9.6883 & 8.9278 & 5.9307 & 6.8826 & 6.5699 & 6.4412 & $1.08 \mathrm{E}-17$ & Down \\
\hline ENSMUSG00000022686 & B3gnt5 & 8.7715 & 9.9054 & 9.2119 & 6.3750 & 7.3837 & 6.6865 & 6.1338 & 2.13E-09 & Down \\
\hline ENSMUSG00000024413 & Npc1 & 10.3509 & 10.7805 & 10.7764 & 8.1749 & 7.6795 & 8.1548 & 6.0209 & $1.55 \mathrm{E}-31$ & Down \\
\hline ENSMUSG00000054901 & Arhgef33 & 8.7177 & 9.4959 & 8.8486 & 4.4594 & 7.2668 & 5.5546 & 5.9614 & 0.002785 & Down \\
\hline ENSMUSG00000028222 & Calb1 & 14.0292 & 14.7406 & 14.2434 & 11.0154 & 12.2761 & 11.8078 & 5.5053 & 1.64E-06 & Down \\
\hline
\end{tabular}

revealed that the mRNAs coexpressed with lncRNAs were mainly linked to the immune system process (biological process, BP), plasma membrane (cellular component, $\mathrm{CC}$ ), and protein binding (molecular function, MF) (Fig. 5A-C). KEGG pathway analysis identified osteoclast differentiation, Staphylococcus aureus infection, and phagosomes as the most enriched pathways (Fig. 5D). 
Fig. 2 Expression patterns of lncRNAs and mRNAs in WT and $\mathrm{NPC} 1^{-1-}$ mice. Volcano plots of DE lncRNAs (A) and mRNAs (B). Blue, red, and black points represent lncRNAs or mRNAs that were downregulated, upregulated, or not significantly different in $\mathrm{NPC1}^{-/-}$ (KO) mice relative to WT mice. Heatmap of DE lncRNAs (C) and mRNAs (D). Red and blue: increased and decreased expression, respectively

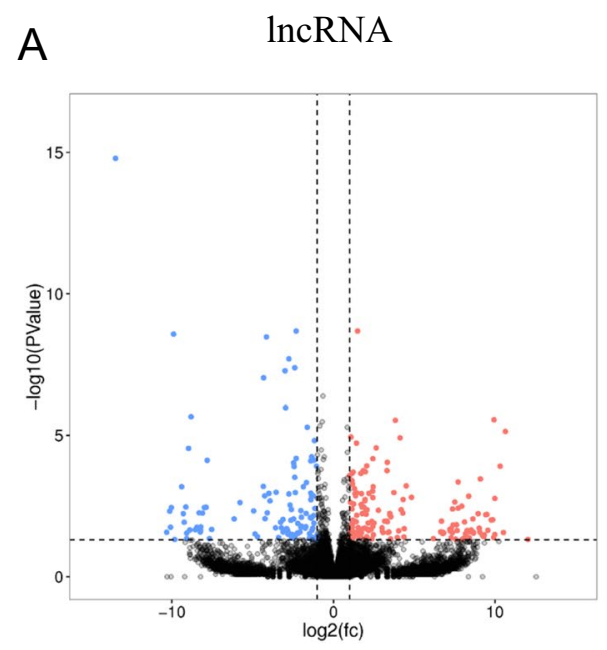

B mRNA

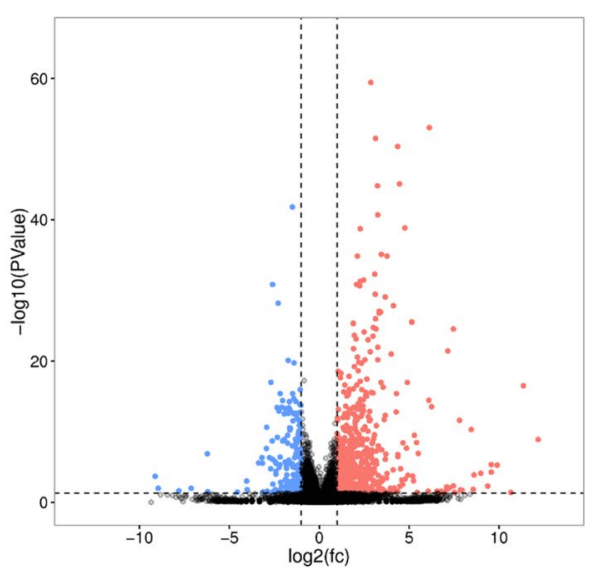

C

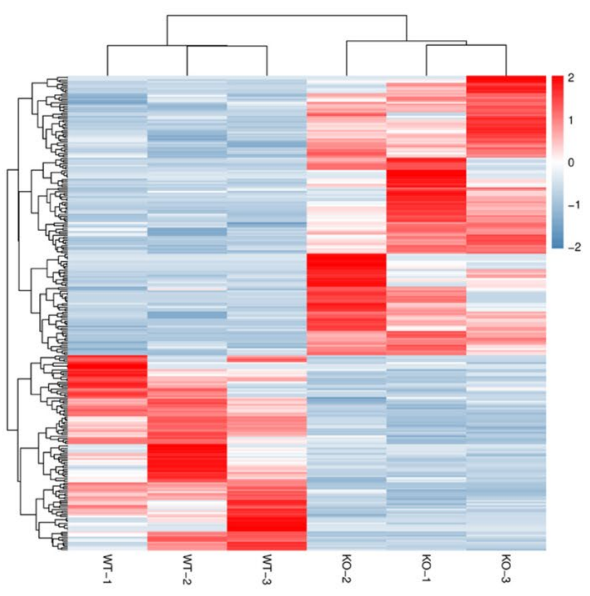

D
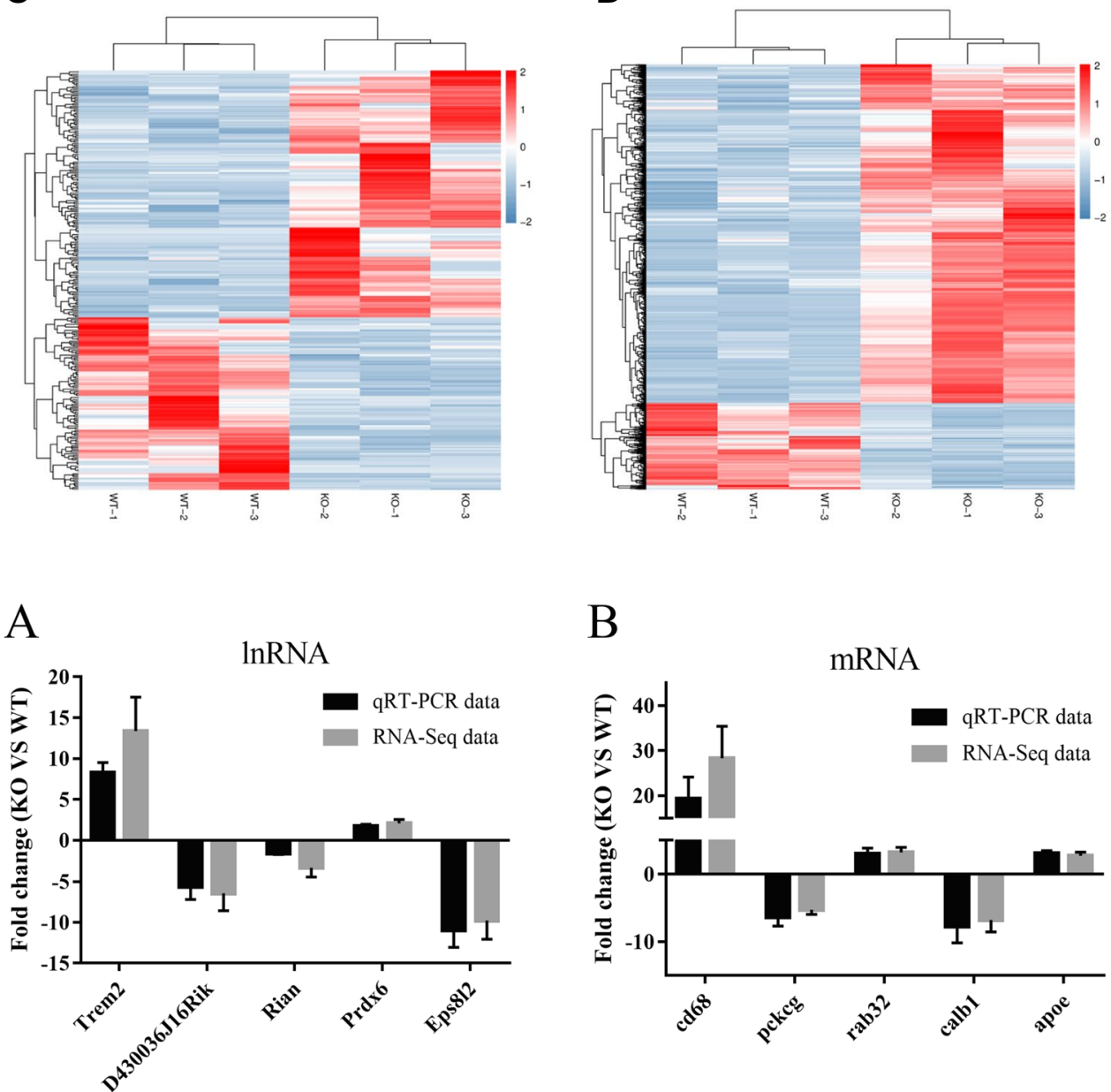

B

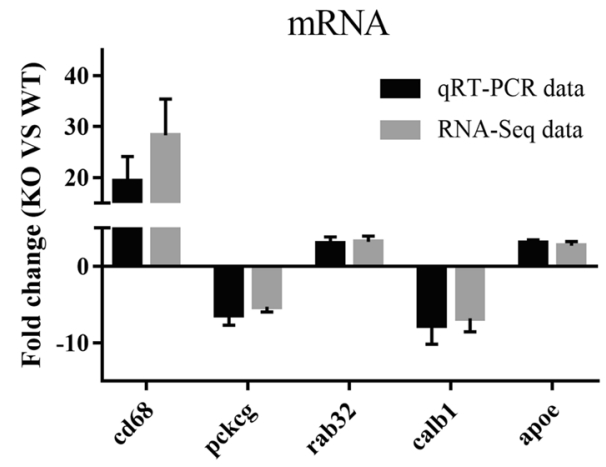

Fig. 3 The differential expression of 5 randomly selected lncRNAs (A) and 5 randomly selected mRNAs (B) was validated by qRT-PCR

\section{NPC1-Related Coexpression Networks in NP-C}

NP-C evolves primarily due to mutations in the NPC1 gene; thus, further investigation of NPC1-lncRNA interactions may significantly enhance our understanding of NP-C. Here, we identified NPC1 coexpression with 7 lncRNAs. Next,
mRNAs coexpressed with the 7 lncRNAs were used to construct a subnetwork (Fig. 6A). Examination of the KEGG pathway annotations for which $p<0.05$ revealed that these genes may be involved in the glycosphingolipid biosynthesis, TGF-beta signaling, protein digestion and absorption, 
Fig. 4 LncRNA-mRNA coexpression network analysis. A Circos diagram of DE lncRNAs and mRNAs. The outermost circle is a schematic of the murine chromosome distribution. The second and third circles (from the outermost circle) represent the chromosomal distribution of DE mRNAs. The fourth and fifth circles represent the chromosomal distribution of DE lncRNAs. Red and green lines indicate up- and downregulation, respectively. Internal connections indicate the top 100 strongest correlations between lncRNAs and mRNAs. B Coexpression network of lysosome (Lys)-related genes and lncRNAs. C Coexpression network of calcium-related genes and lncRNAs. Orange and green nodes represent dysregulated lncRNAs and mRNAs, respectively
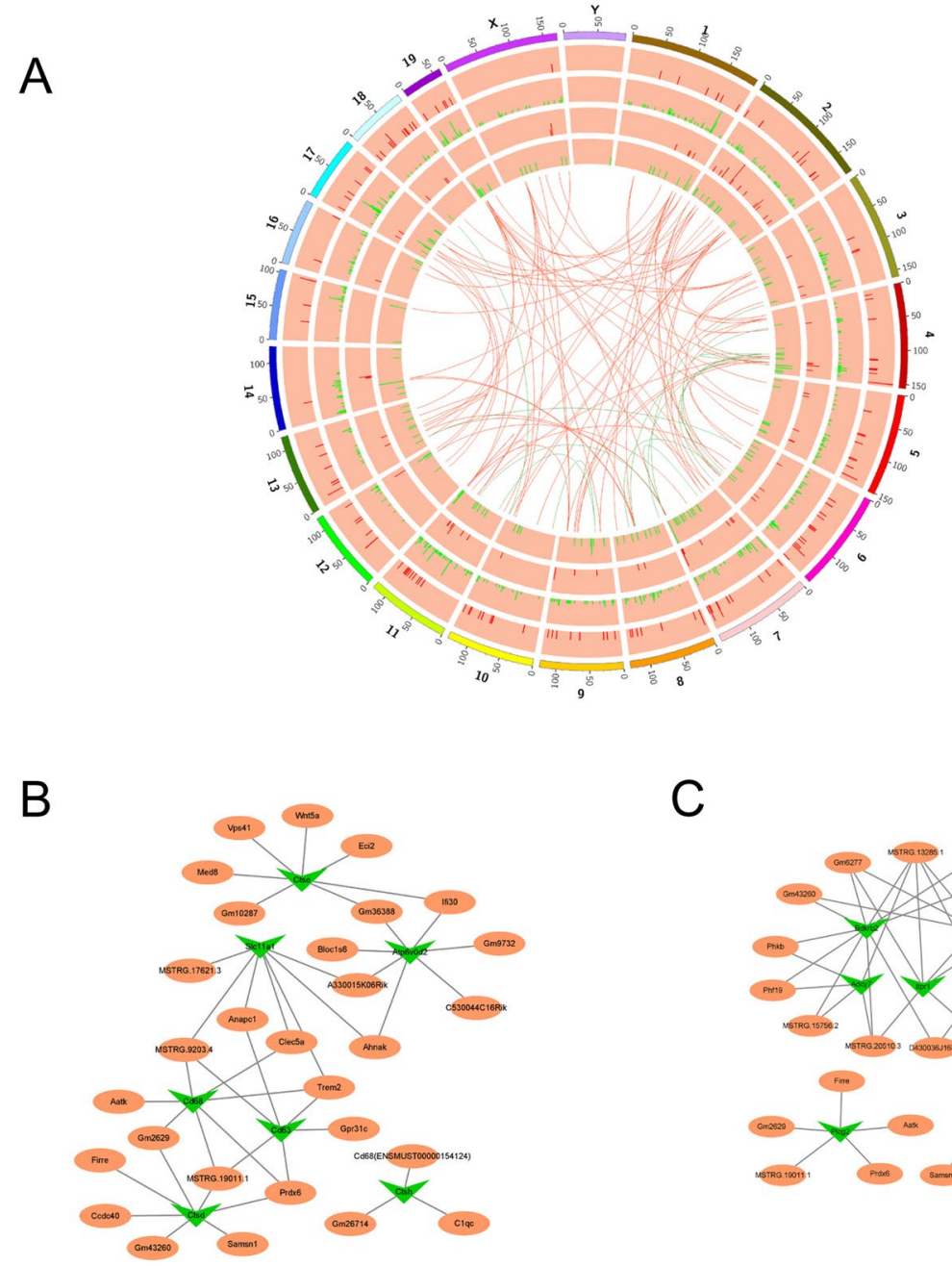

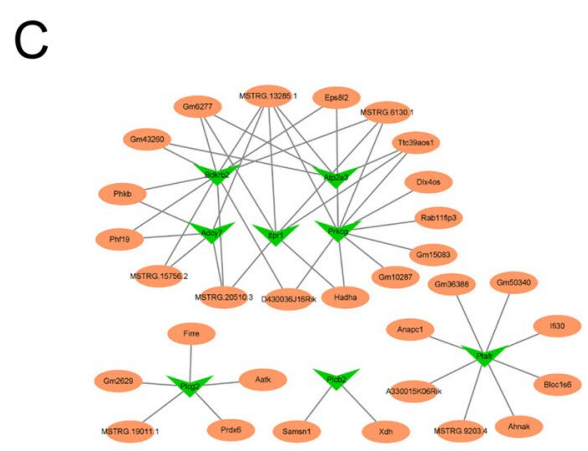

cell adhesion molecule, and neuroactive ligand-receptor interaction pathways (Fig. 6B).

\section{Dysregulated Expression of the IncRNA H19 in NP-C}

Among the top dysregulated lncRNAs (Table 1), we focused on upregulation of the lncRNA $\mathrm{H} 19$ in $\mathrm{NPC1}^{-1-}$ mice. We first validated the dysregulated expression of the lncRNA H19 in cerebellar and liver tissues (Fig. 7A). Increased expression of the lncRNA H19 has been shown to be closely associated with inflammation [32-35]. Next, we explored whether the dysregulated IncRNA H19 was involved in the inflammatory response in vitro. Expression of the lncRNA H19 was significantly increased in skin fibroblasts derived from the $\mathrm{NPC}^{-/-}$mice compared to the WT mice (Fig. 7A). We then used siRNA to successfully knock down H19 in $\mathrm{NPC}^{-/-}$fibroblasts (Fig. 7B). A significantly decreased ROS level was detected in the $\mathrm{NPC}^{-/-}$fibroblasts after knockdown of the lncRNA H19 (Fig. 7C). Moreover, knockdown of the lncRNA H19 reversed the change in viability of the $\mathrm{NPC}^{-/-}$fibroblasts treated with or without LPS, whose viability was similar to that of the WT fibroblasts (Fig. 7D). TNF- $\alpha$, IL-6, and IL-1 $\beta$, as proinflammatory cytokines, promote the inflammatory response, and their expression can be induced by inflammatory activators such as LPS. H19 knockdown attenuated LPS-induced expression of these proinflammatory cytokines in the $\mathrm{NPC}^{-/-}$fibroblasts (Fig. 7E-G). Taken together, these data suggested that lowering $\mathrm{H} 19$ expression could be a strategy to ameliorate oxidative and inflammatory damage in NP-C.

\section{Discussion}

Dysregulated lncRNAs have been suggested to play important roles in the pathological processes of numerous neurodegenerative neurological disorders, particularly neuroinflammation, the modulation of $A \beta$ enrichment/production, synaptic transmission, neurotrophin depletion, and mitochondrial dysfunction [36]. The best-known example of a lncRNA is $\beta$-secretase-1 antisense RNA (BACE1-AS) [12], which drives formation of Alzheimer's disease-implicated 
A

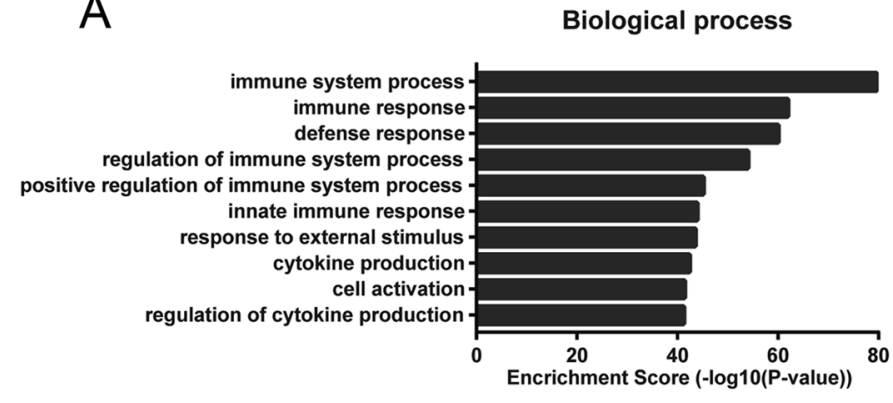

C

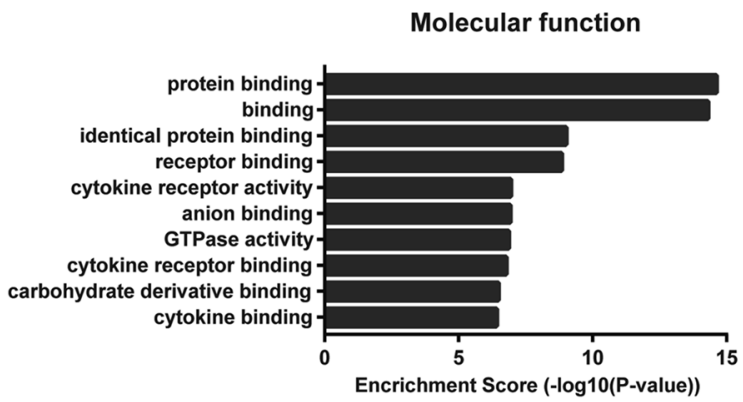

B

Cellular component

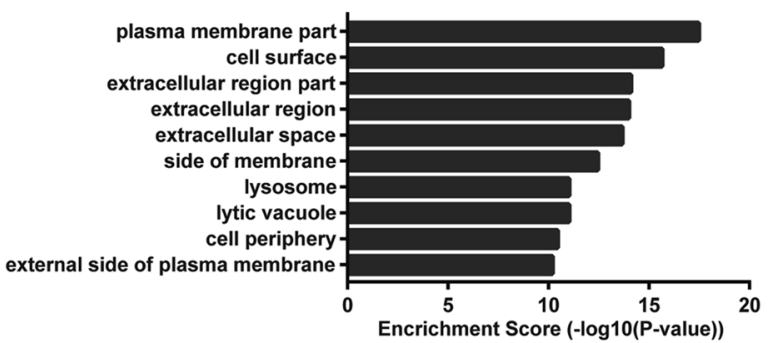

D

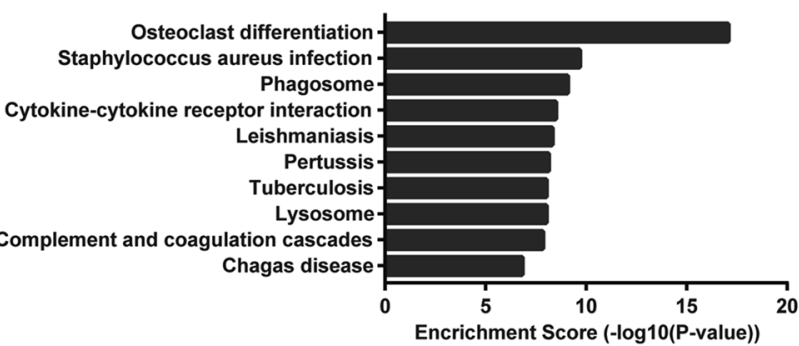

Fig. 5 GO enrichment and KEGG pathway analyses of DE genes in $\mathrm{NPC}^{-1-}$ mice versus WT mice. A BP, B CC, C MF, and D KEGG pathways enriched in the significantly DE genes; the top 10 most significant terms from the enrichment analysis are presented $(p<0.05)$

A

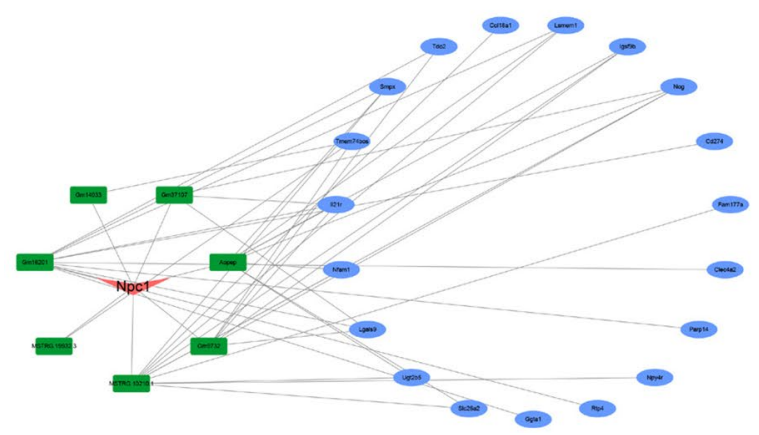

Fig. 6 Construction of a NPC1-related coexpression network. A Green nodes represent lncRNAs that were significantly coexpressed with NPC1. Blue nodes represent coexpressed genes. B KEGG analy-

forms of $\mathrm{A} \beta$ peptides [37]. NP-C is a typical neurodegenerative disorder sometimes referred to as childhood Alzheimer's disease. However, the pathological implications of lncRNAs in NP-C remain undetermined. Cerebellar ataxia, a major hallmark of NP-C disease, is mainly caused by the progressive degeneration of Purkinje cells [6, 8, 38]. Here, we applied RNA-seq analysis to profile the expression patterns of lncRNAs and mRNAs in cerebellar tissue from $\mathrm{NPC}^{-l-}$ mice and uncovered 160 lncRNAs and 682 mRNAs as significantly upregulated and 112 lncRNAs and 174 mRNAs as significantly downregulated compared to their expression in WT littermates $(\mathrm{FC} \geq 2.0, p<0.05)$. The

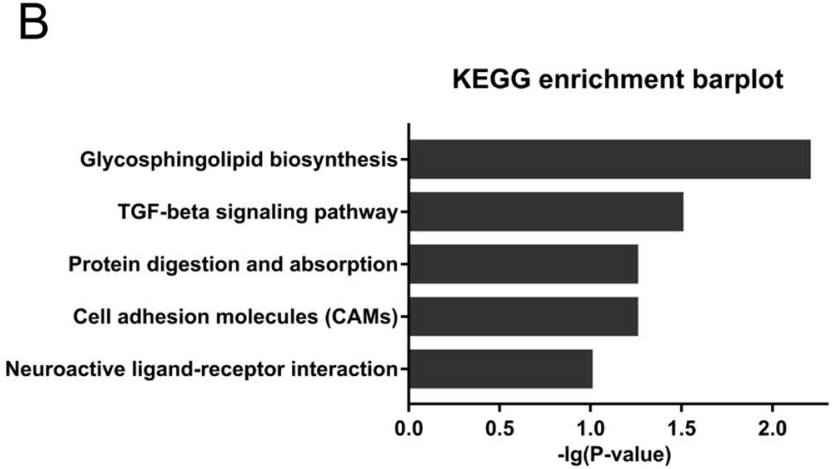

sis indicated that NPC1-lncRNA-coexpressed mRNAs were mainly targeted to the glycosphingolipid biosynthesis pathway

identification of these dysregulated lncRNAs can provide insight into the investigation of novel mechanisms underlying the pathological processes of NP-C.

Given that anomalous lysosomal function and $\mathrm{Ca}^{2+}$ signaling play central roles in NP-C pathology [32, 33], we explored the association between DE IncRNAs and Lys- and calcium-associated genes. We also constructed a NPC1-lncRNA coexpression network and added the mRNAs coexpressed with 7 lncRNAs for pathway annotation analysis. KEGG analysis suggested that genes coexpressed with 7 lncRNAs are mainly enriched in the glycosphingolipid biosynthesis pathway. The accumulation 
Fig. 7 The effect of lncRNA H19 knockdown on oxidative stress. A Expression of H19 in cerebellar, liver and skin fibroblasts of mice. B H19 expression was evaluated after electroporation of H19-siRNA in skin fibroblasts. C ROS generation was detected by CM- $\mathrm{H}_{2}$ DCFDA staining. D The viability of the fibroblasts that treated with or without $100 \mathrm{ng} /$ $\mathrm{mL}$ LPS for $24 \mathrm{~h}$ was detected by CCK- 8 assay. The expression levels of the inflammatory cytokines TNF-a (E), IL-6 (F), and IL- $1 \beta(\mathrm{G})$ after $24 \mathrm{~h}$ of stimulation with $100 \mathrm{ng} / \mathrm{mL}$ LPS were measured by qRTPCR. Data are the mean \pm SEM from three independent experiments. ${ }^{*} p<0.05$ and ${ }^{* *} p<0.01$, compared with the WT group; $\# p<0.05$ and \#\#p $<0.01$, compared with $\mathrm{NPC}^{-/-}+$si-NC group
A
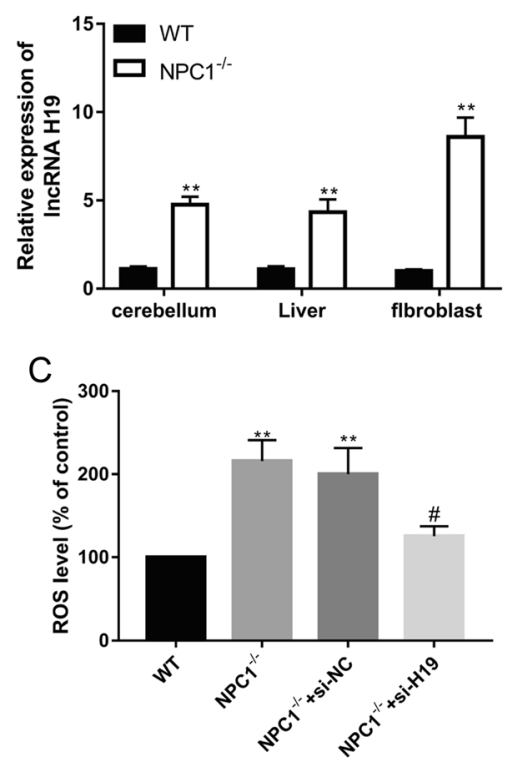

B

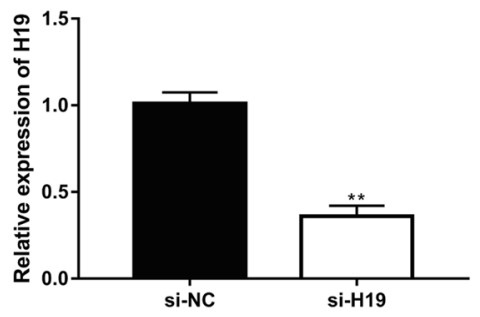

D

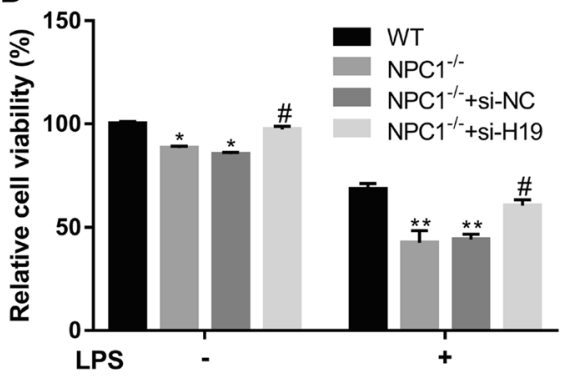

G

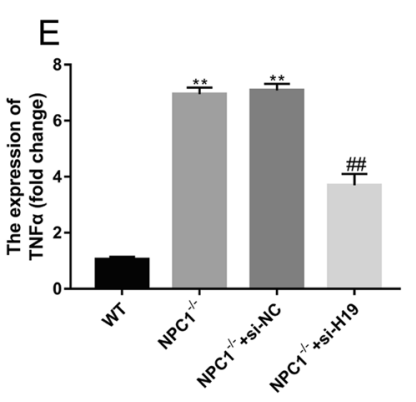

$\mathrm{F}$

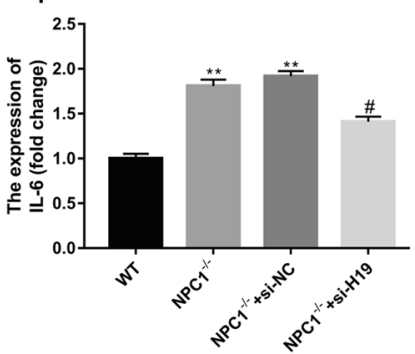

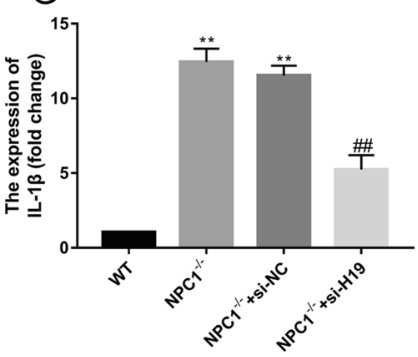

of a massive amount of glycosphingolipid in the neurosystem was demonstrated to be a major feature of NP-C [39]. Currently, miglustat, a glycosphingolipid biosynthesis inhibitor, is the only medication approved for NP-C patients in some areas and countries, including the EU and China [40, 41]. Miglustat administration was found to delay neurological dysfunction onset and extend average life span in NP-C animal models. Moreover, miglustat treatment improved clinical symptoms and quality of life in NP-C patients. However, miglustat was originally developed and applied for Gaucher disease, and its application was extended for NP-C disease. The mechanisms underlying miglustat treatment for NP-C are not clear. Therefore, its clinical application in NP-C patients has not been approved by the FDA in the USA. The dysregulated lncRNAs revealed to be related to impaired glycosphingolipid metabolism due to NPC1 mutation have great potential for the development of novel medications targeting the glycosphingolipid biosynthesis pathway.

GO and KEGG pathway analyses were performed to identify coding genes related to the significantly dysregulated lncRNAs. GO analysis showed that the enrichment of biological processes such as the immune system process, immune response, defense response, and innate immune response, which have been implicated in neurodegenerative diseases and cognitive dysfunction, in these lncRNAs. Neuroinflammation, a common pathological hallmark of most neurodegenerative diseases, influences neuronal development and function [42]. NP-C patients and $\mathrm{NPC}^{-/-}$mice exhibit abnormal mitochondrial function and increased oxidative stress [43, 44]. In our study, we also observed that ROS level significantly increased in the $\mathrm{NPC}^{-1-}$ fibroblasts. Therefore, we suggest that pathological inflammation not only actively contributes to NP-C pathogenesis but also is a potential therapeutic target in NP-C.

The lncRNA H19, which is involved in immune and inflammatory responses, promotes microglia and astrocyte activation under epileptic and normal conditions [45]. In addition, the lncRNA H19 enhances neuroinflammation by driving HDAC1-dependent microglial M1 polarization during ischemic stroke [46]. Here, we found that levels of the IncRNA H19 were significantly upregulated in the cerebellar, liver and skin fibroblasts of $\mathrm{NPC}^{-/-}$mice. Silencing the lncRNA H19 in skin fibroblasts ameliorated the changes in ROS levels and cell viability and inflammatory response induced by LPS, suggesting that inhibition of the lncRNA H19 may improve the pathological features of NP-C disease via inflammatory modulation. 
Some of the other dysregulated lncRNAs that we identified have been reported to be involved in pathological processes in other neurological diseases. The IncRNA Neat1 was found to be significantly upregulated in the caudate nucleus in Huntington's disease [47] and plays an important role in innate immunity [48]. In an ischemic stroke rat model, the lncRNA MIAT could induce the autophagy and apoptosis of neural cells [49]. The lncRNA Rian was decreased in a model of cerebral ischemia-reperfusion injury, and overexpression of the lncRNA Rian significantly reduced infarct size and improved neurological function score [50]. These findings suggest that the aberrant expression of lncRNAs may have similar effects in the above neurological diseases and NP-C.

There are several interesting issues that remain to be addressed. First, we report the profiles of only lncRNAs in the cerebellum, and the expression patterns of IncRNAs in the blood and cerebral spinal fluid in NP-C remain to be determined. Furthermore, it is unclear whether the significantly dysregulated lncRNAs can serve as diagnostic biomarkers for NP-C. Second, we predicted the functions of DE IncRNAs through bioinformatics analysis of mRNAs coexpressed with the IncRNAs; however, it is unclear whether these IncRNAs would modulate expression of the corresponding coding genes in vitro or in vivo. Third, determining the spatiotemporal expression patterns of the DE lncRNAs and mRNAs requires further measurements to more precisely reflect the pathophysiology of NP-C.

\section{Conclusion}

This study has, for the first time, determined the expression patterns of lncRNAs in a NP-C mouse model. Our results show that aberrantly expressed lncRNAs are involved in various pathological processes, especially immune system-related processes, and we demonstrated that the IncRNA H19 was associated with the inflammatory response in vitro. These findings provide new insights into NP-C pathogenesis and unveil novel therapeutic targets.

Supplementary Information The online version contains supplementary material available at https://doi.org/10.1007/s12035-021-02526-3.

Acknowledgements We thank Prof. Jun Zhang for technical assistance in behavioral experiments of mice.

Author Contribution S.H. and Z.Y. designed and supervised the study; S.H., M.R., and T.K. performed behavioral experiments of mice; M.P. and D.G. collected samples and performed qRT-PCR; S.H., Y.L., Y.W., and W.Z. analyzed and discussed the data; S.H. and Z.Y. drafted the manuscript. Z.Y. revised the manuscript.

Funding This work was supported by the grants from the National Natural Science Foundation (NNSF)of China (81170471) and from the Emphasis Basic Medical Scientific Research Project Fund of China (AWS17J007).
Data Availability The RNA-seq raw data are available on Sequence Read Archive (SRA) under the accession number PRJNA682842.

All animal experimental methods performed were conducted in accordance with the ethical standards and procedures of the ethics committee approved by the Army Medical University (Chongqing, China) under permit NO. amuwec20181550.

\section{Declarations}

Conflict of Interest The authors declare no competing interests.

Consent to Participate Not applicable.

Consent for Publication Not applicable.

Open Access This article is licensed under a Creative Commons Attribution 4.0 International License, which permits use, sharing, adaptation, distribution and reproduction in any medium or format, as long as you give appropriate credit to the original author(s) and the source, provide a link to the Creative Commons licence, and indicate if changes were made. The images or other third party material in this article are included in the article's Creative Commons licence, unless indicated otherwise in a credit line to the material. If material is not included in the article's Creative Commons licence and your intended use is not permitted by statutory regulation or exceeds the permitted use, you will need to obtain permission directly from the copyright holder. To view a copy of this licence, visit http://creativecommons.org/licenses/by/4.0/.

\section{References}

1. Vanier MT (2010) Niemann-Pick disease type C. Orphanet J Rare Dis 5:16

2. Wang ML, Motamed M, Infante RE, Abi-Mosleh L, Kwon HJ, Brown MS et al (2010) Identification of surface residues on Niemann-Pick C2 essential for hydrophobic handoff of cholesterol to NPC1 in lysosomes. Cell Metab 12:166-173

3. Infante RE, Radhakrishnan A, Abi-Mosleh L, Kinch LN, Wang ML, Grishin NV et al (2008) Purified NPC1 protein: II. Localization of sterol binding to a 240 -amino acid soluble luminal loop. J Biol Chem 283:1064-1075

4. Millard EE, Gale SE, Dudley N, Zhang J, Schaffer JE, Ory DS (2005) The sterol-sensing domain of the Niemann-Pick C1 (NPC1) protein regulates trafficking of low density lipoprotein cholesterol. J Biol Chem 280:28581-28590

5. Vanier MT (2015) Complex lipid trafficking in Niemann-Pick disease type C. J Inherit Metab Dis 38:187-199

6. Elrick MJ, Pacheco CD, Yu T, Dadgar N, Shakkottai VG, Ware C et al (2010) Conditional Niemann-Pick $\mathrm{C}$ mice demonstrate cell autonomous Purkinje cell neurodegeneration. Hum Mol Genet 19:837-847

7. Walkley SU, Suzuki K (2004) Consequences of NPC1 and NPC2 loss of function in mammalian neurons. Biochim Biophys Acta 1685:48-62

8. Ko DC, Milenkovic L, Beier SM, Manuel H, Buchanan J, Scott MP (2005) Cell-autonomous death of cerebellar purkinje neurons with autophagy in Niemann-Pick type C disease. PLoS Genet 1:81-95

9. Hammond N, Munkacsi AB, Sturley SL (2019) The complexity of a monogenic neurodegenerative disease: More than two decades of therapeutic driven research into Niemann-Pick type $\mathrm{C}$ disease. Biochim Biophys Acta Mol Cell Biol Lipids 1864:1109-1123

10. Mercer TR, Dinger ME, Mattick JS (2009) Long non-coding RNAs: insights into functions. Nat Rev Genet 10:155-159 
11. Wu YY, Kuo HC (2020) Functional roles and networks of non-coding RNAs in the pathogenesis of neurodegenerative diseases. J Biomed Sci 27:49

12. Faghihi MA, Modarresi F, Khalil AM, Wood DE, Sahagan BG, Morgan TE et al (2008) Expression of a noncoding RNA is elevated in Alzheimer's disease and drives rapid feed-forward regulation of beta-secretase. Nat Med 14:723-730

13. Magistri M, Velmeshev D, Makhmutova M, Faghihi MA (2015) Transcriptomics profiling of Alzheimer's disease reveal neurovascular defects, altered amyloid- $\beta$ homeostasis, and deregulated expression of long noncoding RNAs. J Alzheimers Dis 48:647-665

14. Wang Y, Zhao X, Ju W, Flory M, Zhong J, Jiang S et al (2015) Genome-wide differential expression of synaptic long noncoding RNAs in autism spectrum disorder. Transl Psychiatry 5:e660

15. Ni Y, Huang H, Chen Y, Cao M, Zhou H, Zhang Y (2017) Investigation of long non-coding RNA expression profiles in the substantia nigra of Parkinson's disease. Cell Mol Neurobiol 37:329-338

16. Sunwoo JS, Lee ST, Im W, Lee M, Byun JI, Jung KH et al (2017) Altered expression of the long noncoding RNA NEAT1 in Huntington's disease. Mol Neurobiol 54:1577-1586

17. Feng L, Liao YT, He JC, Xie CL, Chen SY, Fan HH et al (2018) Plasma long non-coding RNA BACE1 as a novel biomarker for diagnosis of Alzheimer disease. BMC Neurol 18:4

18. Modarresi F, Faghihi MA, Lopez-Toledano MA, Fatemi RP, Magistri M, Brothers SP et al (2012) Inhibition of natural antisense transcripts in vivo results in gene-specific transcriptional upregulation. Nat Biotechnol 30:453-459

19. Langmade SJ, Gale SE, Frolov A, Mohri I, Suzuki K, Mellon SH et al (2006) Pregnane X receptor (PXR) activation: a mechanism for neuroprotection in a mouse model of Niemann-Pick $\mathrm{C}$ disease. Proc Natl Acad Sci U S A 103:13807-13812

20. Marshall CA, Watkins-Chow DE, Palladino G, Deutsch G, Chandran K, Pavan WJ et al (2018) In Niemann-Pick C1 mouse models, glial-only expression of the normal gene extends survival much further than do changes in genetic background or treatment with hydroxypropyl-beta-cyclodextrin. Gene 643:117-123

21. Guyenet SJ, Furrer SA, Damian VM, Baughan TD, La Spada AR, Garden GA (2010) A simple composite phenotype scoring system for evaluating mouse models of cerebellar ataxia. J Vis Exp 39:1787

22. Liu W, Wang Z, Wang C, Ai Z (2019) Long non-coding RNA MIAT promotes papillary thyroid cancer progression through upregulating LASP1. Cancer Cell Int 19:194

23. Pertea M, Pertea GM, Antonescu CM, Chang TC, Mendell JT, Salzberg SL (2015) StringTie enables improved reconstruction of a transcriptome from RNA-seq reads. Nat Biotechnol 33:290-295

24. Love MI, Huber W, Anders S (2014) Moderated estimation of fold change and dispersion for RNA-seq data with DESeq2. Genome Biol 15:550

25. Pujana MA, Han JD, Starita LM, Stevens KN, Tewari M, Ahn JS et al (2007) Network modeling links breast cancer susceptibility and centrosome dysfunction. Nat Genet 39:1338-1349

26. Seluanov A, Vaidya A, Gorbunova V (2010) Establishing primary adult fibroblast cultures from rodents. J Vis Exp 44:2033

27. Loftus SK, Morris JA, Carstea ED, Gu JZ, Cummings C, Brown A et al (1997) Murine model of Niemann-Pick C disease: mutation in a cholesterol homeostasis gene. Science 277:232-235

28. Kim D, Langmead B, Salzberg SL (2015) HISAT: a fast spliced aligner with low memory requirements. Nat Methods 12:357-360

29. Sun L, Luo H, Bu D, Zhao G, Yu K, Zhang C et al (2013) Utilizing sequence intrinsic composition to classify protein-coding and long non-coding transcripts. Nucleic Acids Res 41:e166

30. Kang YJ, Yang DC, Kong L, Hou M, Meng YQ, Wei L et al (2017) CPC2: a fast and accurate coding potential calculator based on sequence intrinsic features. Nucleic Acids Res 45:W12-W16

31. Guttman M, Rinn JL (2012) Modular regulatory principles of large non-coding RNAs. Nature 482:339-346
32. Lloyd-Evans E, Morgan AJ, He X, Smith DA, Elliot-Smith E, Sillence DJ et al (2008) Niemann-Pick disease type C1 is a sphingosine storage disease that causes deregulation of lysosomal calcium. Nat Med 14:1247-1255

33. Tiscione SA, Vivas O, Ginsburg KS, Bers DM, Ory DS, Santana LF et al (2019) Disease-associated mutations in Niemann-Pick type $\mathrm{C} 1$ alter ER calcium signaling and neuronal plasticity. J Cell Biol 218:4141-4156

34. Wang B, Suen CW, Ma H, Wang Y, Kong L, Qin D et al (2020) The Roles of H19 in Regulating Inflammation and Aging. Front Immunol 11:579687

35. Shi X, Wei YT, Li H, Jiang T, Zheng XL, Yin K et al (2020) Long non-coding RNA H19 in atherosclerosis: what role? Mol Med 26:72

36. Li L, Zhuang Y, Zhao X, Li X (2018) Long non-coding RNA in neuronal development and neurological disorders. Front Genet 9:744

37. Kang MJ, Abdelmohsen K, Hutchison ER, Mitchell SJ, Grammatikakis I, Guo R et al (2014) HuD regulates coding and noncoding RNA to induce $\mathrm{APP} \rightarrow \mathrm{A} \beta$ processing. Cell Rep 7:1401-1409

38. Vite CH, Bagel JH, Swain GP, Prociuk M, Sikora TU, Stein VM et al (2015) Intracisternal cyclodextrin prevents cerebellar dysfunction and Purkinje cell death in feline Niemann-Pick type $\mathrm{C} 1$ disease. Sci Transl Med 7:226r-276r

39. Te Vruchte D, Lloyd-Evans E, Veldman RJ, Neville DC, Dwek RA, Platt FM et al (2004) Accumulation of glycosphingolipids in Niemann-Pick C disease disrupts endosomal transport. J Biol Chem 279:26167-26175

40. Patterson MC, Vecchio D, Prady H, Abel L, Wraith JE (2007) Miglustat for treatment of Niemann-Pick $\mathrm{C}$ disease: a randomised controlled study. Lancet Neurol 6:765-772

41. Te VD, Lloyd-Evans E, Veldman RJ, Neville DC, Dwek RA, Platt FM et al (2004) Accumulation of glycosphingolipids in Niemann-Pick C disease disrupts endosomal transport. J Biol Chem 279:26167-26175

42. Chen WW, Zhang X, Huang WJ (2016) Role of neuroinflammation in neurodegenerative diseases (Review). Mol Med Rep 13:3391-3396

43. Torres S, Matías N, Baulies A, Nuñez S, Alarcon-Vila C, Martinez L et al (2017) Mitochondrial GSH replenishment as a potential therapeutic approach for Niemann Pick type C disease. Redox Biol 11:60-72

44. Yambire KF, Fernandez-Mosquera L, Steinfeld R, Mühle C, Ikonen E, Milosevic I et al (2019) Mitochondrial biogenesis is transcriptionally repressed in lysosomal lipid storage diseases. Elife 8:e39598

45. Han CL, Ge M, Liu YP, Zhao XM, Wang KL, Chen N et al (2018) LncRNA H19 contributes to hippocampal glial cell activation via JAK/STAT signaling in a rat model of temporal lobe epilepsy. $\mathrm{J}$ Neuroinflammation 15:103

46. Wang J, Zhao H, Fan Z, Li G, Ma Q, Tao Z et al (2017) Long noncoding RNA H19 promotes neuroinflammation in ischemic stroke by driving histone deacetylase 1-dependent M1 microglial polarization. Stroke 48:2211-2221

47. Johnson R (2012) Long non-coding RNAs in Huntington's disease neurodegeneration. Neurobiol Dis 46:245-254

48. Zhang P, Cao L, Zhou R, Yang X, Wu M (2019) The lncRNA Neat1 promotes activation of inflammasomes in macrophages. Nat Commun 10:1495

49. Guo X, Wang Y, Zheng D, Cheng X, Sun Y (2021) LncRNA-MIAT promotes neural cell autophagy and apoptosis in ischemic stroke by up-regulating REDD1. Brain Res 1763:147436

50. Yao P, Li YL, Chen Y, Shen W, Wu KY, Xu WH (2020) Overexpression of long non-coding RNA Rian attenuates cell apoptosis from cerebral ischemia-reperfusion injury via Rian/miR-144-3p/GATA3 signaling. Gene 737:144411

Publisher's Note Springer Nature remains neutral with regard to jurisdictional claims in published maps and institutional affiliations. 